\title{
The 3rd International EuroFIR Congress 2009: European Food Composition Data for Better Diet, Nutrition and Food Quality
}

\author{
P Finglas $^{1}$, E Weichselbaum ${ }^{2}$ and JL Buttriss ${ }^{2}$ \\ ${ }^{1}$ Institute of Food Research, Norwich, UK and ${ }^{2}$ British Nutrition Foundation, London, UK \\ European Journal of Clinical Nutrition (2010) 64, S1-S3; \\ doi:10.1038/ejen.2010.201
}

EuroFIR (short for 'European Food Information Resource Network of Excellence') was a five-and-a-half-year project funded by the European Commission's Research Directorate General under the 'Food Quality and Safety Priority' of the Sixth Framework Programme for Research and Technological Development. EuroFIR is a partnership involving 48 universities, research institutes and small-to-medium-sized enterprises from 27 European countries, comprising almost 200 researchers and $\mathrm{PhD}$ students. European Union funding for the project ended in June 2010, but work will continue within the recently established legal entity EuroFIR AISBL (Association Internationale Sans But Lucratif), an international, non-profit, member-based association of food composition data researchers, expert users and stakeholders, based in Belgium.

EuroFIR's main objective was to provide the first comprehensive pan-European food information resource, using state-of-the-art database linking, which provides online access to various authoritative national sources of food composition data established for particular European countries, providing detailed information on nutrients and bioactive compounds with potential health benefits. The need for this European food information resource has, for some time, been recognised as an underpinning requirement essential to the improvement of pan-European food and health research and the development of European public health policy. It has also become central to the development and implementation of the European regulation on nutrition and health claims made on foods (EC No. 1924/2006, for more details see Buttriss and Benelam (2010) and Kiely et al. (2010), in this supplement), to the development of an associated

Correspondence: P Finglas, Institute of Food Research, Norwich, UK. E-mail: paul.finglas@bbsrc.ac.uk nutrient profiling model and for risk assessment in tandem with food consumption surveys.

One of EuroFIR's key achievements has been the establishment of a new legal entity, EuroFIR AISBL (http://www. eurofir.eu/eurofir_aisbl), by eight of the current EuroFIR partner organisations. EuroFIR AISBL is a member-based, international, non-profit association of food composition data compilers, expert users and stakeholders, based in Belgium. The primary mission of EuroFIR AISBL is the development, publication and exploitation of food composition data and accompanying information in order to support and underpin research into food quality, food safety, and diet and health in Europe. EuroFIR AISBL provides a key interface between the national food database compiler organisations and their national funders and stakeholders, including a network of laboratories producing the data, and users of food composition data from industry, academia and regulatory affairs. It also promotes and develops appropriate quality assurance and traceability principles, facilitating the implementation of relevant international standards.

EuroFIR AISBL offers a single and unique food information resource to academia, industry, public sector funding bodies and regulators, as well as to individuals such as researchers, students and dietitians, who are all welcome to join the association as a member (find more information on membership at http://www.eurofir.eu). Owing to its close links with nutritionists, dietitians and public health professionals, EuroFIR AISBL can support pan-European nutritional and epidemiological studies. It offers state-of-the-art knowledge about methods of analysis for a diverse range of nutrients and non-nutritive bioactive compounds with putative health benefits, expert knowledge about food databases and innovative data interfaces, as well as state-of-the-art solutions for various data applications in a broad spectrum of food and health topics. 


\section{EuroFIR's achievements presented at the 3rd International EuroFIR Congress}

This special issue of the European Journal of Clinical Nutrition is dedicated to the publication of papers following presentations at the 3rd International EuroFIR Congress 2009: European Food Composition Data for Better Diet, Nutrition and Food Quality, held on 8-10 September 2009 in Vienna, Austria. The congress was jointly organised by the British Nutrition Foundation, UK; the Institute of Food Research, UK; and the Institute of Nutritional Sciences, University of Vienna, Austria.

The congress brought together more than 150 delegates from 30 countries, including EuroFIR consortium members and other key stakeholders and users of food composition data. The programme included 40 presentations over seven sessions covering the following topics:

- using food composition data to address current public health and nutrition issues;

- major public health/nutrition education tools and software that utilise food composition data and databases;

- new food composition data/databases-the need for quality standards;

- new or novel methods of analyses for nutrients and nonnutrient bioactive compounds in foods and supplements;

- new data on traditional and ethnic foods in Europe;

- delivering food composition data through emerging interfaces for dietary assessment and health applications.

The plenary lectures given at the congress included summaries of the progress made within EuroFIR. These were complemented by plenary contributions from outside of the EuroFIR network and poster presentations were available to view.

A key objective of EuroFIR was the harmonisation and standardisation of work on food composition data across Europe. Development of the EuroFIR databank system required that individual databases were established according to common principles, and that data in national online databases are presented, with their metadata, in a uniform manner (see http://www.eurofir.eu/?q=node/8). To ensure these prerequisites, EuroFIR has contributed to the drafting of new recommendations in the form of a new European Standard on Food Data within the framework of the European Committee for Standardisation (CEN, see Becker (2010) in this supplement). The paper by Oliveira et al. (2010) in this supplement reports on the strategy used and progress made for extending the quality management system of the Portuguese National Institute of Health (INSA) to the Portuguese food composition database, in alignment with these EuroFIR guidelines, and thus presents an example of how the guidelines may be applied.

Food description forms an essential attribute of the EuroFIR standard (http://www.eurofir.eu/food_information/ food_identification_and_description). EuroFIR uses LanguaL (Langua Alimentaria: http://www.langual.org) as its primary tool in food description. LanguaL is a structured system for describing food, and is well suited to the systematic retrieval of data from numerical databases, making linking of different food composition databases and data sets possible (Møller et al., 2007). By the end of the project, more than 26000 foods in 27 national food composition databases, together with around 2300 foods in four specialised data sets, have been linked using LanguaL and therefore form the basis of the European Food Data Platform. The paper by Ireland and Møller (2010) in this supplement describes the main features of LanguaL and the work being carried out within EuroFIR and EuroFIR AISBL.

A paper by Pakkala et al. (2010) describes the interchange of food composition data that happens through the EuroFIR Web Services interface. The interface allows the national partners to implement their food composition database systems into the EuroFIR Web Services interface using methods and software suitable for the local computer environment. Pakkala et al. (2010) also describe the specifically constructed EuroFIR search facility (eSearch available at http://esearch. eurofir.org). This facility was designed for end users in order to make food composition data from various international food composition databases more readily accessible. It uses a specifically designed Food Data Query Language to compile queries and a specifically designed data interchange format (the EuroFIR Food Data Transport Package) in XML (Extensible Markup Language) for query responses.

As part of EuroFIR's objectives on the identification and provision of new information and data for components, nutrients and for non-nutrient compounds with putative health effects, EuroFIR has designed and implemented a process for the identification, prioritisation, collection and analysis of both traditional and ethnic foods, using a common methodology for European countries.

EuroFIR's traditional foods work package aimed to study the composition of traditional foods from different countries, in order to preserve these aspects of European culture, and also to use the new data to enrich and improve dietary habits across the whole continent (Trichopoulou et al., 2006). Five traditional foods have been selected from each of 13 European countries (Austria, Belgium, Bulgaria, Denmark, Germany, Greece, Iceland, Italy, Lithuania, Poland, Portugal, Spain and Turkey) and sent to appropriately quality-verified laboratories for selected nutrient analyses. Dr Helena Soares Costa, who has been the traditional foods work package leader since September 2006, presented the results of the work package at the congress. More information on the methods used for selecting and analysing traditional foods, as well as the main outcomes, is presented in the paper by Costa et al. (2010) in this supplement.

EuroFIR's ethnic foods work package aimed to provide new and reliable data on the nutritional composition of up to five commonly consumed ethnic foods or recipes from seven selected European countries, and Israel, for inclusion in national food composition databases. The results of this work package were presented by Dr Santosh Khokhar from 
the University of Leeds and the main findings, including the results from the nutritional analyses, are presented in the paper by Khokhar et al. (2010) in this special issue.

The EuroFIR network has established EuroFIR eBASIS (BioActive Substances in Foods Information System), which is an on-line database that uniquely combines food composition and biological effects data for plant-based bioactive compounds, hosted at http://ebasis.eurofir.org. It is a compilation of critically evaluated data extracted from peer-reviewed literature and consists of two compatible databases providing extensive food composition data and biological effects data for plant bioactives, as well as an up-to-date list of the major food plants consumed in Europe. EuroFIR eBASIS covers multiple bioactive compound classes and major European food plants, with data sourced from quality-assessed, peer-reviewed primary publications (Gry et al., 2007). Two of the main users of EuroFIR eBASIS are the European Food Safety Authority and the food industry. Potential uses of the database for these users have been identified, including the substantiation of nutrition and health claims, calculations of exposure to bioactive compounds and the development of new food products. In this supplement, the paper by Kiely et al. (2010) evaluates the EuroFIR eBASIS biological effects database in the preparation and evaluation of health claims dossiers.

Training opportunities within EuroFIR were designed to build capacity and spread excellence in the field of food composition. EuroFIR's training programme covered individual exchanges, specifically designed to enable EuroFIR PhD students to visit other partners and attend conferences and specialised workshops (for example, training in LanguaL for indexing foods, documentation and quality systems for the compilers). It also includes general training in food composition (the FoodComp Courses and EuroFIR e-learning modules). A paper by Gurinovic et al. (2010) in this issue presents an inventory of the status of food composition databases and the training needs of compilers in non-EuroFIR countries in Central and Eastern Europe, the Middle East and North Africa. It also presents the capacity development achieved in these regions through EuroFIR and other network collaborations.

Many other interesting topics that are related to food composition data and databases are presented in this special issue. These include papers on the following topics: the contribution of ethnic foods to total nutrient intake in a Bedouin minority in Israel; a CEN standard on food data; disaggregating composite food codes in the UK National Diet and Nutrition Survey; the contribution of the French Observatory of Food Quality to nutritional quality and labelling of breakfast cereals; glycaemic index values in the Finnish food composition database; web-based eHealth applications with reference to food composition data; full value documentation in the Czech food composition database; EURRECA: development of tools to improve the alignment of micronutrient recommendations; identification of the 100 richest dietary sources of polyphenols-an application of the Phenol-Explorer database; food composition data in health communication; biogenic amines in fermented foods; FLABEL_Food Labelling to Advance Better Education for Life; and evaluation of food consumption and dietary patterns in Spain.

We are grateful to the organisers of the 3rd International EuroFIR Congress for making it such a success and to the authors and referees of the articles contained in this special issue for their time and expertise.

\section{Conflict of interest}

The authors declare no conflict of interest.

\section{References}

Becker W (2010). Towards a CEN Standard on food data. Eur J Clin Nutr 64(Suppl 3), S49-S52.

Buttriss JL, Benelam B (2010). Nutrition and health claims: the role of food composition data. Eur J Clin Nutr 64(Suppl 3), S8-S13.

Costa HS, Vasilopoulou E, Trichopoulou A, Finglas P (2010). New nutritional data on traditional foods for European food composition databases. Eur J Clin Nutr 64(Suppl 3), S73-S81.

Gry J, Black L, Eriksen FD, Pilegaard K, Plumb J, Rhodes $M$ et al. (2007). EuroFIR-BASIS-A combined composition and biological activity database for bioactive compounds in plant-based foods. Trends Food Sci Tech 18, 434-444.

Gurinović M, Witthöft CM, Tepšić J, Ranić M, Hulshof PJM, Hollman PC et al. (2010). Capacity development in food composition database management and nutritional research and education in Central and Eastern European, Middle Eastern and North African countries. Eur J Clin Nutr 64(Suppl 3), S134-S138.

Ireland JD, Møller A (2010). LanguaL Food Description: a Learning Process. Eur J Clin Nutr 64(Suppl 3), S44-S48.

Khokhar S, Marletta L, Shahar DR, Farre R, Ireland JD, Jansen-van der Vliet M et al. (2010). New food composition data on selected ethnic foods consumed in Europe. Eur J Clin Nutr 64(Suppl 3), S82-S87.

Kiely M, Black LJ, Plumb J, Kroon PA, Hollman PC, Larsen JC et al. (2010). EuroFIR eBASIS: application for health claims submissions and evaluations. Eur J Clin Nutr 64(Suppl 3), S101-S107.

Møller A, Unwin I, Becker W, Ireland J (2007). EuroFIR's food databank systems for nutrients and bioactives. Trends Food Sci Tech 18, 428-433.

Oliveira LM, Castanheira IP, Dantas MA, Porto AA, Calhau MA (2010). Portuguese Food Composition Database Quality Management System. Eur J Clin Nutr 64(Suppl 3), S53-S57.

Pakkala H, Christensen T, Martínez de Victoria I, Presser K, Kadvan A (2010). Harmonized information exchange between decentralized food composition database systems. Eur J Clin Nutr 64(Suppl 3), S58-S63.

Trichopoulou A, Vasilopoulou E, Georga K, Soukara S, Dillis V (2006). Traditional foods: Why and how to sustain them. Trends Food Sci Tech 17, 498-504. 